\title{
Assessment of Three Multiple Cuff Blood Pressure Devices
}

\author{
Jiri Jilek \\ Carditech \\ Culver City \\ California \\ USA \\ jjilek30@gmail.com
}

\author{
Milan Stork \\ Department of Applied Electronics and \\ Telecommunications/RICE \\ University of West Bohemia \\ Plzen, Czech Republic \\ stork@kae.zcu.cz
}

\begin{abstract}
Shortcomings of single cuff blood pressure devices have led to research of devices with more than one cuff for improved accuracy. We assessed two devices that use two cuffs and one device that uses three cuffs. The devices with two cuffs use one occluding arm cuff and one cuff that detects pulses that pass past the occluding cuff. The device with three cuffs uses pulse delay time. The advantages and the shortcomings of the devices are discussed in this paper.
\end{abstract}

Keywords - Blood pressure; cuff pulse; dual cuff; triple cuff; pulse detection; delay time; device validations

\section{INTRODUCTION}

Traditional noninvasive measurement of blood pressure (BP) consists of a sphygmomanometer with a cuff placed on patient's arm and a stethoscope. The stethoscope is applied to the brachial artery distally to the cuff and Korotkoff sounds are observed. Systolic blood pressure (SBP) is the point of the first sound and diastolic pressure (DBP) is the point of sound disappearance. This method is still used and it is considered to be the gold standard [1].

More than a generation ago, automatic electronic BP devices have been introduced. Single cuff automatic blood pressure (BP) monitors are the most prevalent BP devices on the market today. Most BP monitors use arm cuffs but wrist cuff devices are also popular. Single cuff devices usually use cuff pressure (CP) and arterial pressure pulse data acquired from the cuff during cuff deflation. Fig. 1 shows pressure pulse amplitudes (PA) during gradual cuff deflation. Maximal pulse amplitude (MAX PA) is used for determination of systolic (SBP) and diastolic (DBP) pressures. The method is called characteristic ratio method. The amplitude ratios are based on statistically predetermined ratios of MAX PA and amplitudes on the high CP side for SBP and on the low CP side for DBP. This method is commonly called the oscillometric method.

Milan Stork's participation was supported by Department of Applied Electronics and Telecommunications, University of West Bohemia, Plzen, Czech Republic and by the Ministry of Education, Youth and Sports of the Czech Republic under the RICE - New Technologies and Concepts for Smart Industrial Systems, project No. LO1607 and the Internal Grant Agency of University of West Bohemia in Plzen, the project SGS-2018-001

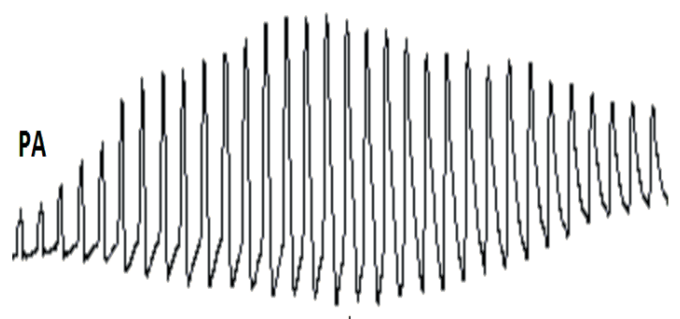

$$
\text { HIGHCP MAXPA LOWCP }
$$

Figure 1. Arterial pulse waveforms during gradual cuff deflation. $\mathrm{CP}$ is cuff pressure and PA is pulse amplitude

This method eliminated the need of pulse detection distally of the cuff (the stethoscope), but this innovation came at the cost of decreased accuracy and reliability of measurement [2]. Single cuff BP devices need to be validated according to a validation protocol. Manual BP method is used for validations.

The needs for increased accuracy and reliability of automatic BP devices have led to research and development of several devices that use more than one cuff [3]. The methods used in three of such devices are assessed in this paper. We concentrated on the function of the additional cuffs that are used for pulse detection distally to the occluding cuff. All three methods use personal computers for control, data acquisition and data processing.

\section{METHODS}

\section{A. Method \#1}

This method [4] uses a standard arm cuff $(13 \times 24$ $\mathrm{cm})$ and a small cuff $(3 \times 6 \mathrm{~cm})$ placed inside the distal side of the arm cuff over the brachial artery (Fig. 2). The instrumentation consists of pressure transducers, analog and digital circuits, and a computer with specially developed software.

The arm cuff functions as the brachial artery occluding cuff and the pulsations in the pulse detection cuff are used to determine the points of SBP and DBP 

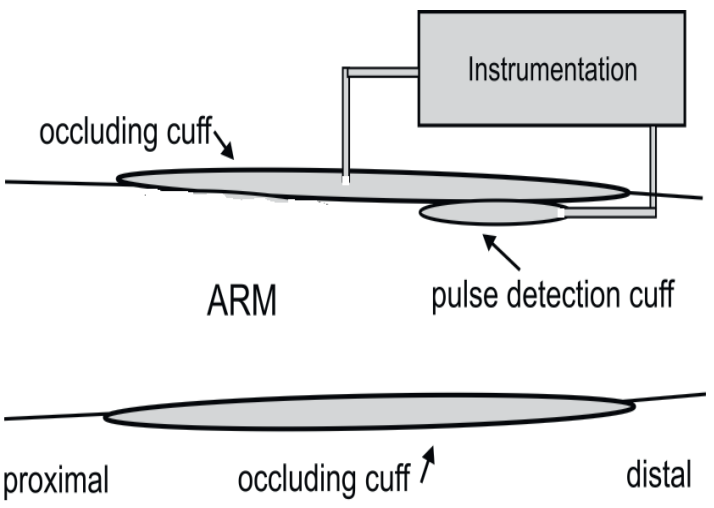

Figure 2. The two cuff method with occluding cuff, pulse detection cuff, and instrumentation

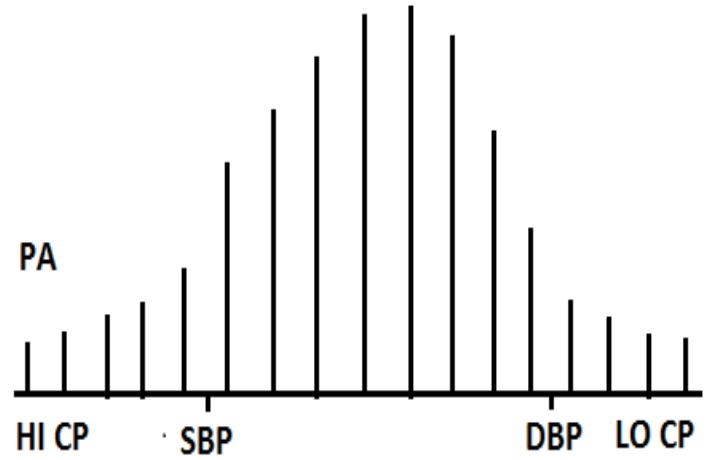

Figure 3. Picket fence representation of arterial pulse amplitudes (PA) during gradual cuff pressure $(\mathrm{CP})$ deflation

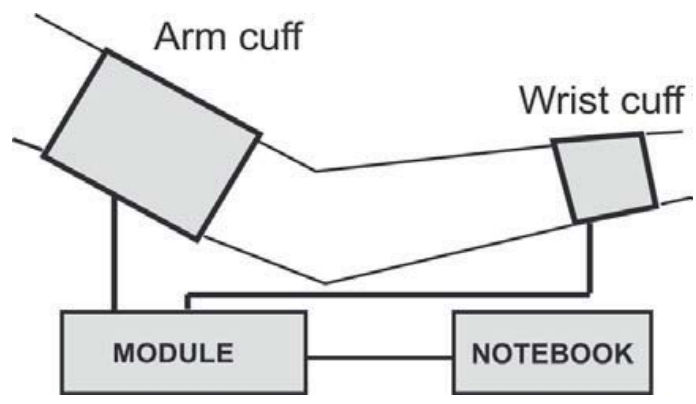

Figure 4. The dual cuff system with arm cuff, wrist cuff, the instrumentation module, and the notebook computer

The cuff pressures and their superimposed pulsations are converted to voltage by pressure transducers and processed by the computer. Dedicated software is used to evaluate pulsations in the small cuff. The picket fence representation of the pulse amplitudes (PA) is shown in Fig. 3. The value of SBP is determined as the point of sudden increase of PA. The value of DBP is determined as the point of sudden decrease of PA.

\section{B. Method \#2}

This method [5] uses two commercial cuffs of different sizes. The occluding cuff is placed on the upper arm and the pulse detecting cuff is placed on the wrist (Fig. 4). The arm cuff size is $14 \times 24 \mathrm{~cm}$ and the wrist cuff size is $6 \times 11 \mathrm{~cm}$.

All functions of the system are controlled by special software in the notebook computer. The notebook communicates with instrumentation module via USB. The module contains the pneumatic and electronic circuits.

The wrist cuff function is similar to the auscultatory BP determination. The wrist cuff is inflated to about DBP level during the cuff inflation procedure and it is held at that level until the end of the BP test. The wrist cuff pressure level is not critical but the wrist cuff amplitudes are optimal at DBP level. The arm cuff is inflated to pressure above the expected SBP level and then deflated slowly. Picket fence representation of wrist cuff pulse amplitudes is shown in Fig. 5. When cuff pressure is lowered to SBP level, the wrist cuff pulses are detected in the wrist cuff. As the arm cuff pressure (CP) is lowered further, the pulse amplitudes rapidly increase. The SBP value can be determined from the onset of PAs.

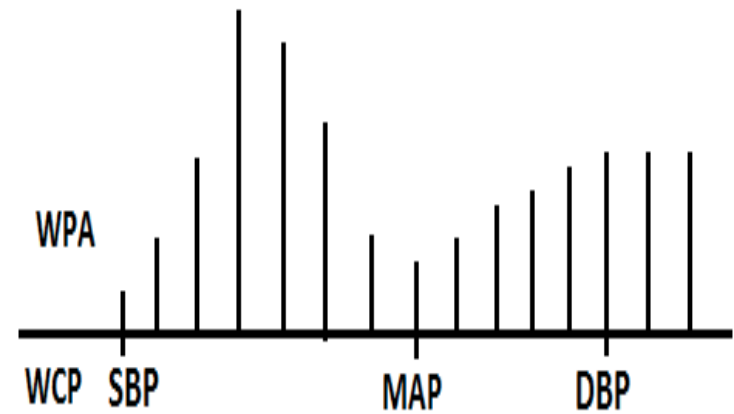

Figure 5. Picket fence representation of wrist cuff pulse amplitudes (WPA) during gradual cuff deflation. Wrist cuff pressure WCP is held at near DBP pressure. WPA are wrist cuff pulse amplitudes

Wrist pulse amplitudes quickly reach peak value and then decrease until they reach low point. This point corresponds to mean arterial pressure (MAP) .

The determination of DBP relies on subtler waveform changes. The wrist cuff pulse amplitudes increase from the point of MAP until they reach a plateau. At this point (see Fig. 5) the corresponding arm cuff pressure is taken as the DBP value.

\section{Method \#3}

Fujikawa et al [6] described a pulse delay technique with triple cuff. Fig. 6 shows the triple cuff arrangement. The main cuff serves as a standard arm occluding cuff, the second cuff is the pulse detecting cuff and the third cuff serves as a "notch enhancing" cuff. While the occluding cuff is a standard cuff wrapped around the arm, the other two cuffs can be better described as air bags because of their much smaller size. The small cuffs are not wrap-around type cuffs. The pulses in the second cuff are used to determine the points of SBP and DBP. 

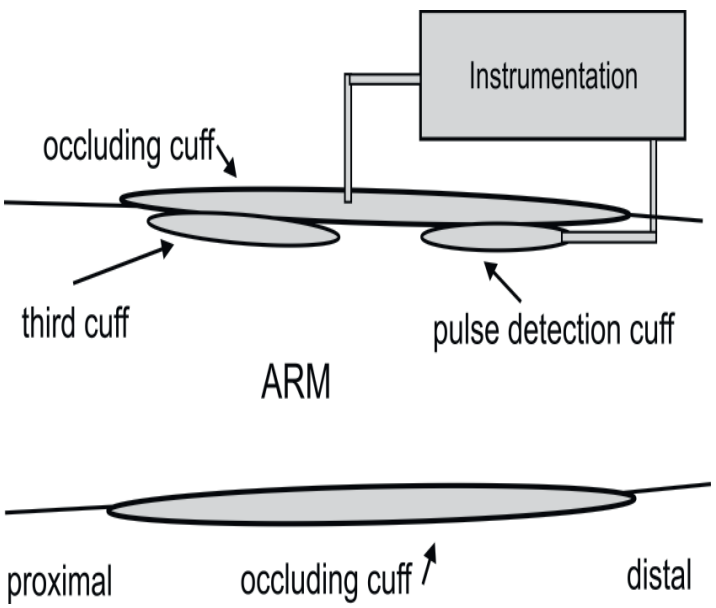

Figure 6. Triple cuff configuration. Pulse detection cuff is located at the distal side of the occluding cuff

The delay time is measured between the proximal pulse upstroke onset and the distal upstroke onset. Fig. 7 shows the delay time changes with time. At occluding cuff pressures above SBP the upstroke delay time is short. When occluding cuff pressure is lowered to the SBP level, the delay time abruptly increases. This increase is registered as the point of SBP. As cuff pressure is gradually lowered the delay time decreases until the level of DBP is reached. At this time the delay ceases to decrease and the cuff pressure at this point is registered as DBP.

Instrumentation unit and a personal computer were used for data acquisition and processing. Specially designed software automatically takes care of inflation and deflation of the cuffs, the acquisition and processing of cuff data, and display of results.

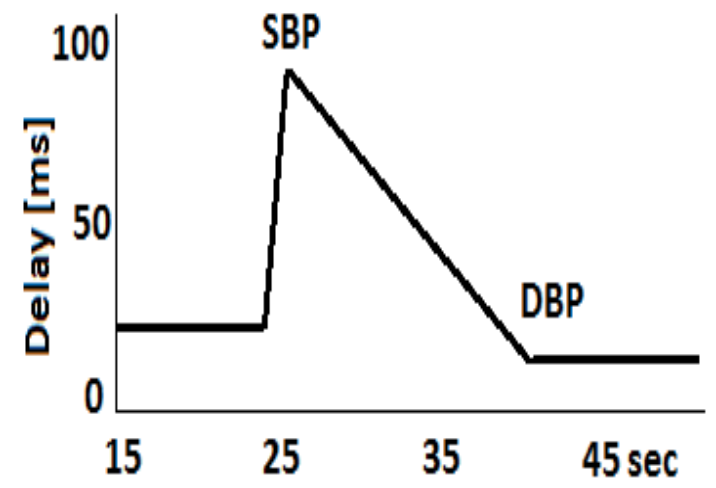

Figure 7. Graph of delay time during occluding cuff gradual deflation. The SBP is at the point of maximal delay time and DBP is at the beginning of delay time plateau

\section{RESULTS}

Method \#1 was compared with auscultation. Mean difference for SBP was $0.42 \mathrm{mmHg}$ and $\mathrm{SD}$ was 2.9 $\mathrm{mmHg}$. Mean difference for DBP was $1.2 \mathrm{mmHg}$ and $\mathrm{SD}$ was $3.5 \mathrm{mmH}$.
Method \#2 SBP and DBP values were compared with the single cuff characteristic ratio method. This method was used on the same data set because the occlusion cuff data are automatically available. The ratio value for SBP was 0.5 and the ratio value for DBP was 0.76. Mean difference for SBP was 5.9 $\mathrm{mmHg}$ and SD was $9.62 \mathrm{mmHg}$. The difference for DBP was $3.2 \mathrm{mmHg}$ and SD was $4.52 \mathrm{mmHg}$.

Method \#3 BP values were compared with values obtained with the auscultatory method. Mean difference for SBP was $2.53 \mathrm{mmHg}$ and SD was 2.62 $\mathrm{mmHg}$. Mean difference for DBP was $3.9 \mathrm{mmHg}$ and $\mathrm{SD}$ was $5.76 \mathrm{mmHg}$.

\section{DISCUSSION}

All three methods described above attempt to improve accuracy that may be lacking in the single cuff methods. The accuracy improvement is accomplished by employing additional cuff or cuffs. The disadvantage of all three methods is that additional cuffs and electronics are necessary. The small cuff used by method \#1 is used to produce arterial pulse envelope (Fig. 3) that produces abrupt amplitude changes at SBP and DBP points. These changes facilitate more accurate SBP and DBP determination. The problem with method \#1 could be potential corruption of pulses with incorrect placement of the pulse detecting cuff. There have been methods in the past that used contact microphone or ultrasound probe under the occluding cuff. These methods are no longer used because of the problems of probe placement.

Method \#2 uses two commercial cuffs that are easily obtained. The pulse detecting wrist cuff is completely removed from the occluding cuff. The placement of the wrist cuff is not critical and it can be accomplished by anybody. The advantage of this approach is that the determination of SBP is more reliable because it is easy to detect first appearance of the pulses in the wrist cuff. In addition to the determination of SBP and DBP values, this method can determine the values of mean arterial pressure (MAP). MAP is a useful hemodynamic variable which is used to determine peripheral vascular resistance.

Method \#3 uses three cuffs and the placement of the two small cuffs are important. Similarly to the method \#1 they must be placed over the brachial artery. Cuff misplacement can cause errors. The time delay method used here is new and it is difficult to assess how practical it may be in clinical environment.

There may be an important application of an accurate multiple cuff BP method. At the present time electronic single cuff devices are validated using the manual auscultatory method [7]. The auscultatory method has its own accuracy problems that are well recognized [1]. In addition, the auscultatory raw data is not available - only the results of BP measurements are available. Multiple cuff computerized methods can store raw data that can be analyzed and stored for later use. Validations of BP devices could be accomplished more reliably and the stored data could be verified. 


\section{CONCLUSION AND FUTURE WORK}

The multiple cuff methods assessed in this paper attempt to improve accuracy of noninvasive blood pressure measurement. They could be useful in research or clinical environment where accuracy is more important than in routine blood pressure screening. Better validation methods of single cuff devices could also be accomplished. Eventually, a computerized BP system should replace the manual BP method as the new gold standard. More studies will be necessary to ascertain which environment is the most suitable for multiple cuff BP devices.

\section{REFERENCES}

[1] D.W. Jones, L. J. Appel, S. G. Sheps , E. J. Roccella, C. Lenfant. "Measuring Blood Pressure Accurately". JAMA 2003; 289:1027-1030.
[2] J. Jilek, T. Fukushima. "Oscillometric Blood Pressure Measurement: The Methodology, Some Observations, and Suggestions". Biomed Instrum Technol 2005; 39:237-241.

[3] A. Avolio, G. Parati. "Improvements on cuff measurement of arterial pressure: more cuffs!" J Hypertens 2013: 31: 251252

[4] O. Tochikubo, J. Watanabe, K. Hanada, E. Myiajima, K. Kimura. "A New Double Cuff Sphygmomanometer For Accurate Blood Pressure Measurement", Hypertens Res 2001; 24: 353-357.

[5] J. Jilek, M. Stork. "Wrist Cuff Emulates Auscultation in Dual-Cuff Noninvasive Blood Pressure System". Proc Int Conf AE2012, Plzen, Czech Republic 2012.

[6] T. Fujikawa, O. Tochikubo, T. Sugano, S. Umemura "Accuracy of the pulse delay time technique with triple cuff for objective indirect blood pressure measurement". J Hypertens, 2013; 31: 278-286

[7] E. O.Brien, N. Atkins. "Validation and Reliability of Blood Pressure Monitors". Ed. W. White, Blood Pressure Monitoring in Cardiovascular Medicine and Therapeutics Humana Press, Inc. U.S.A. 2007: 97-132. 